\title{
Achieving Robotically Peeled Lettuce
}

\author{
Josie Hughes, Luca Scimeca, Ioana Ifrim, Perla Maiolino and Fumiya Iida
}

\begin{abstract}
Robotic technologies are being increasingly applied to agriculture, in particular to harvesting. Some types of produce such as iceberg lettuce require additional processing after harvesting in order to satisfy the needs of the end-user or customer. Lettuce must have its outer leaves removed, a task that is currently performed manually. The leaf removal task represents a challenging vision and manipulation problem: the lettuce is in a random pose on a flat surface, from which the outermost leaves must be removed quickly and without causing damage. This letter presents a novel vision pipeline and suction removal system that enables robotic lettuce leaf removal. A suction nozzle and control procedure are used for the removal itself, relying on the orientation estimation and stem detection provided by the vision pipeline. To the best of the author's knowledge, this is the first robotic lettuce leaf peeling system.
\end{abstract}

Index Terms-Agricultural automation, computer vision for automation, grippers and other end-effectors.

\section{INTRODUCTION}

$\mathbf{T}$ HE automation of agricultural systems presents many challenges due to the extreme variability in the environment e.g. varying light, terrain and weather conditions, unlike constrained industrial or lab environments. As such, the successful application of robotics systems to agriculture remains limited. However there is a growing need to develop automated robotic solutions due to the increasing demand for food, sub-optimal climate conditions and decreasing labor availability [1]. The majority of existing robotic agriculture research has focused on developing harvesting systems [2]-[5]. However, often there is a crucial, labor-intensive post-processing step required after harvesting to produce supermarket ready produce [6], [7].

Previous work has seen the development of vision, learning and manipulation solutions for lettuce harvesting [8]. However, supermarkets require the complete removal of one or two loose outer-leaves from the stem after the harvesting process, with no bruising, damage or 'browning' to the lettuce [9], [10] (Fig. 1). This is a challenging task requiring dexterous manipulation and robust vision as the leaves are fragile, tear easily and have limited identifiable features.

Manipulation of soft produce is challenging; dexterity is required and the produce must not be damaged. Although soft

This work was funded by the UK Agriculture and Horticulture Development Board (Project CP 172) and the EPSRC CDT in Sensor Technologies (Grant EP/L015889/1)

${ }^{1}$ All authors are with the Machine Intelligence Laboratory, University of Cambridge, Trumpington Street, CB2 1PZ, Cambridge UK, Email jaeh2@cam.ac.uk 1s769@cam.ac.uk, ii242@cam.ac.uk, fi224@cam.ac.uk.

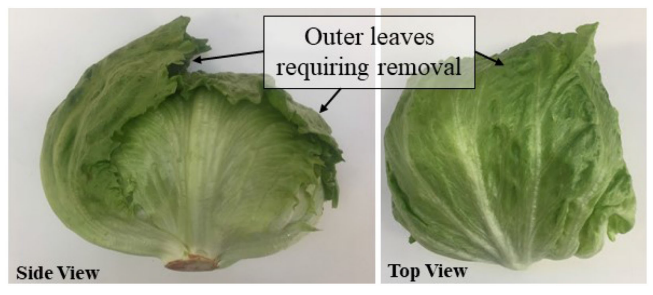

Fig. 1. Iceberg lettuce before processing showing the two outer leaves which require removal.

robotics has significant potential for such applications [11], current solutions provide limited dexterity and require complex control [12]. The vision problem is also challenging, especially when using only 2D stereo cameras. The variability in produce and the lack of rotational varying features makes lettuce a difficult object to interact with, and means existing approaches cannot be used [3]-[5], [13].

The problem addressed is this work is the removal of outer leaves, typically two, from a harvested lettuce. The produce, placed in an unknown pose on a flat environment, should not be damaged by the peeling process. The proposed system uses a single vacuum suction point to grab a leaf and remove it from the main body of the lettuce by tearing. Suction is a method which has been applied successfully in other agriculture applications and reduces the complexity of the required control [14], [15]. This process has been be optimized to maximize successful tearing.

To address this problem, firstly computer vision must be used to locate and determine the pose of the lettuce. A novel machine vision pipeline has been created for orientation detection which uses only a 2D web camera. The pipeline is robust, and unlike existing approaches it does not require depth information from RGB-D, TOF cameras or stereo vision [3]-[5], [13]. The lettuce must then be manipulated into a known state with the outer leaves exposed. The nozzle diameter, material properties, location and trajectory of motion must then be optimized for successful leaf removal.

In this letter the specific methods and implementation are given in Section II, with corresponding results in Section III. Complete testing and demonstration of the leaf removal system is shown in Section IV, concluding with a discussion and review of the work presented.

\section{METHODS \& IMPLEMENTATION}

\section{A. Vision \& Detection}

For a lettuce placed in a unknown location within a work space, machine vision must be used to determine the location 


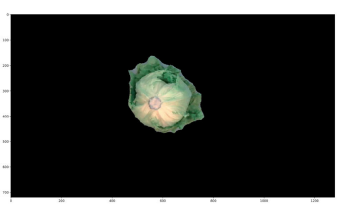

(a)

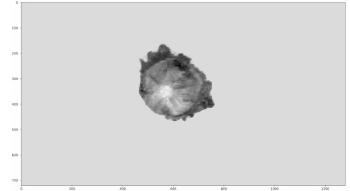

(b)
Fig. 2. (a) Example masked frame, after segmentation. (b) Example lettuce under the A-channel of the LAB color space, showing homogeneous stem pixels.

and orientation of the lettuce. The former is possible through simple lettuce image segmentation. We propose a novel method for retrieving pose by detecting the lettuce stem. This method is necessary as the lack of depth information makes previously explored solutions unsuitable [16]. Moreover, existing approaches for $2 \mathrm{D}$ images use color thresholding techniques which are not applicable as the stem hue and saturation can vary significantly depending on freshness and growing conditions of the lettuce [2], [17].

a) Lettuce Segmentation and Position Estimation: The vision system uses a single Carl Zeiss Tezzar HD 1080p camera placed above the work space, where it is assumed a single lettuce is within the field of vision. The background is assumed to be distinguishable in color to the lettuce, thus, a combination of color-based thresholding and binary cleaning is used for robustness.

The approach adopted uses the HSV (Hue, Saturation and Value) format of the retrieved frame. Two noisy, binary masks are computed by thresholding the Hue channel for pixels $30<p^{h}<100$ and the Saturation channel, for pixels $p^{s}>50$. These are combined with an element-wise AND operation. Hue thresholding is empirically set to retrieve pixels with a color pigmentation within the typical lettuce range. The Saturation channel thresholding removes the false positives due to no luminosity and discarding the brightness channel provides robustness to varying lighting conditions.

In the second step, we clean the mask by applying a morphological dilation followed by an erosion, with a circular disk small enough to connect only nearby pixels ( $25 \mathrm{px}$ in radius). Finally, we find the contour of the largest connected binary area in the mask, set the enclosed pixels to 1, and extract the lettuce from the original frame by masking it with the computed mask (Fig. 2(a)). The center of the connected binary area in the final mask is assumed to be the center position of the lettuce.

b) Stem Detection: Stem detection is the first step towards retrieving a reliable orientation estimation of a lettuce. Due to radical variations in hue and saturation depending on the freshness of the produce, stem detection can not be performed using simple thresholding. A sliding window is used to perform a sequential search of the area within the detected lettuce. In the search we attempt to identify a set of features unique to the stem. The image is converted into the LAB color space, such that value of the pixels within the stem area will be homogeneous across different hues, saturation and lighting conditions. The A-channel spans from a minimum where pixels partake a green coloring, to a maximum where they show a red hue. As the stem never has the same coloring as the green outer leaves, the

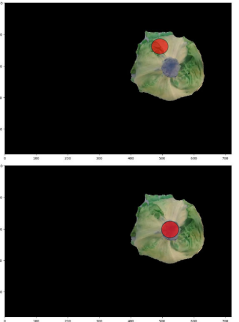

(a)

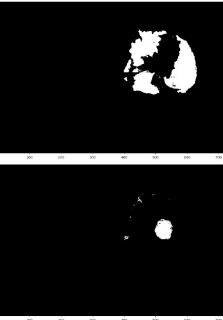

(b)

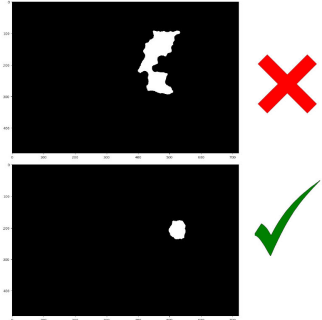

(c)
Fig. 3. The stem detection process: (a) search amongst selected pixels in the lettuce, (b) thresholding on the value of the looked up pixel, (c) cleaning the image. The stem candidate is accepted only after validation.

A-channel is suitable for the stem detection. Fig. 2(b) shows the A-channel of a detected masked lettuce picture after the LAB conversion.

The stem detection process starts by finding the expected size of the stem within the lettuce. Let $\mathbf{M}$ be the previously computed binary mask. We first find the area of the lettuce $a_{l}$ as the sum of the pixels in the mask. The radius of the circle whose area is the same as the detected lettuce is $r_{l}=\sqrt{\frac{a_{l}}{\pi}}$. Experimentally, the stem diameter has been determined to be $\approx \frac{r_{l}}{5}$, with an average error of $\pm \frac{r_{l}}{12}$.

The stem look-up is performed by searching pixels, sliding left to right and top to bottom within the area where the lettuce is contained. The stride length, $\iota$, is tuned to a value small enough to fall within the expected area of the stem multiple times before the end of the search, i.e.: $\iota=\frac{r_{s}^{e}}{4}$, where $r_{s}^{e}$ is the expected radius of the stem in the lettuce.

Let $\mathbf{A}$ be the A-channel of the image in the LAB color space. For each set of indexes $(i, j), \mathbf{A}$ is thresholded around the value of $\mathbf{A}_{\mathbf{i}, \mathbf{j}}$, thus creating an initial masked window $\mathbf{M}^{\mathbf{f}}{ }^{\prime}$, where we compute the element in position $(k, m)$ as:

$$
\mathbf{M}_{\mathbf{k}, \mathbf{m}}^{\mathbf{f}^{\prime}}= \begin{cases}1 & \text { if } \mathbf{A}_{\mathbf{k}, \mathbf{m}}>\mathbf{A}_{\mathbf{i}, \mathbf{j}}-\epsilon \text { and } \mathbf{A}_{\mathbf{k}, \mathbf{m}}<\mathbf{A}_{\mathbf{i}, \mathbf{j}}+\epsilon \\ 0 & \text { otherwise }\end{cases}
$$

The $\epsilon$ determines the strength of the thresholding around the currently inspected pixel (Fig. 3(b)), where $0<\epsilon<256$. We empirically set $\epsilon=7$, to ensure homogeneity amongst the thresholded pixels.

The binary image is de-noised by performing an opening morphological operation with a disk of $\frac{r_{l}}{8}$ followed by a closing operation with a disk of disk radius $=\frac{r_{l}}{3}$. The parameters were chosen to cope with the expected radius of a stem. Finally, we retrieve the largest binary area contour and set the enclosed pixels to 1 (Fig. 3(c)). We will refer to the clean mask as matrix $\mathbf{M}^{\mathbf{f}}$. The binary area in $\mathbf{M}^{\mathbf{f}}$ is an estimate of the stem location in the image. To validate the stem we have devised four unit test: vicinity, comparative area, solidity and elongation.

For vicinity, we test if the $(x, y)$ coordinate of the binary area found after the cleaning operation is within a distance equal to the expected radius of the stem. In the comparative area test, we retrieve the expected area of the stem as $a_{s}^{e}=r_{s}^{e 2} \pi$, and validate the binary area $a_{s}$ if $\left|a_{s}-a_{s}^{e}\right|<a_{s}^{e}$. In the third test, solidity is a measure of the convexity of the found area. Here we compute 
the solidity sol, as:

$$
\text { sol }=\frac{a_{s}}{\Phi\left(\breve{C}^{s}\right)} \text { and } s o l \in\left\{x \in \mathbb{R}_{+} \mid x<1\right\}
$$

where $\breve{C}^{s}$ is the contour of the binary area in $\mathbf{M}^{\mathbf{f}}$ and $\Phi\left(\breve{C}^{s}\right)$ is the area of the convex hull surrounding the contour $\breve{C}^{s}$. In general we expect a stem to be approximately round (i.e. $s o l=1$ ), so we reject a candidate if $s o l<0.7$.

In the fourth and last test we require the stem to be circular, by assuming convexity, we can test this by $\frac{\Psi\left(\breve{C}^{s}\right)}{\psi\left(\breve{C}^{s}\right)}<\zeta$ where $\Psi\left(\breve{C}^{s}\right)$ is the length of the major axis in $\breve{C}^{s}, \Psi\left(\breve{C}^{s}\right)$ the length of its minor axis, and $\zeta$ calibrates the accepted error elongation margin (here $\zeta=1.2$, to ensure approximately round binary areas).

Once all tests are passed, the $(x, y)$ position in the frame will be returned as the location of the lettuce stem (Fig. 3). In this process, almost all tunable parameters are based on the previously found radius of the lettuce. Therefore, assuming that produce can be reliably detected this is a generic process for stem detection.

c) Lettuce Orientation Estimation: Given the shape, a lettuce can be found in one of two poses: stem facing downward or facing up. In the case where the stem cannot be found, an action can be taken to flip the lettuce over. If the stem is found, it is possible to find the pose as a 3D vector starting from the stem and pointing outward, towards the front of the lettuce. This vector is given as:

$$
\left[\begin{array}{c}
p_{l[0]}-p_{s[0]} \\
p_{l[1]}-p_{s[1]} \\
h_{l}^{e}
\end{array}\right]
$$

where $p_{l}$ is the estimated center of the lettuce, $p_{s}$ the position of the stem and $h_{l}^{e}$ the expected lettuce height. As the proposed solution is based on single 2D images, the height of the lettuce is expected to be the average computed height in a data-set of 10 iceberg lettuces $\left(\mu_{l}=111.27 \mathrm{~mm} \pm 3.51 \mathrm{~mm}\right)$. The radius can not be used due to the elliptical shape of lettuce. The leaf removal process is tolerant to changes in height, and thus height errors were found to have a negligible impact on the peeling process.

\section{B. Lettuce Orientation}

By applying a horizontal force and rolling the lettuce, a lettuce can be oriented with the outer-leaf on top and with minimal risk of damage (Fig. 4). Modelling the lettuce as an ellipse, when a force is applied the lettuce will roll and then stop at a stable point where the centre of mass is at the lowest point, i.e. the top and bottom side of the lettuce. This corresponds to angles $\theta$, between the stem and the normal, of $\theta \approx 0$ and $\theta \approx 180$ (Fig. 5). The optimum distance to apply this force over to roll the lettuce such that it is in the top position $(\theta \approx 0)$ must be found.

\section{Leaf Removal}

4) Nozzle Design: 3D printed circular nozzles of varying inner diameters have been used, with a $3 \mathrm{~mm}$ lip around the nozzle

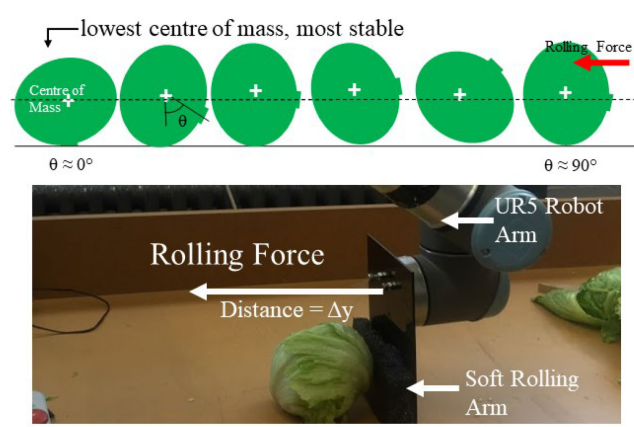

Fig. 4. Diagrammatic representation of the lettuce (top) and method for rolling the lettuce using a soft pad attached to a UR5 robot arm (bottom).
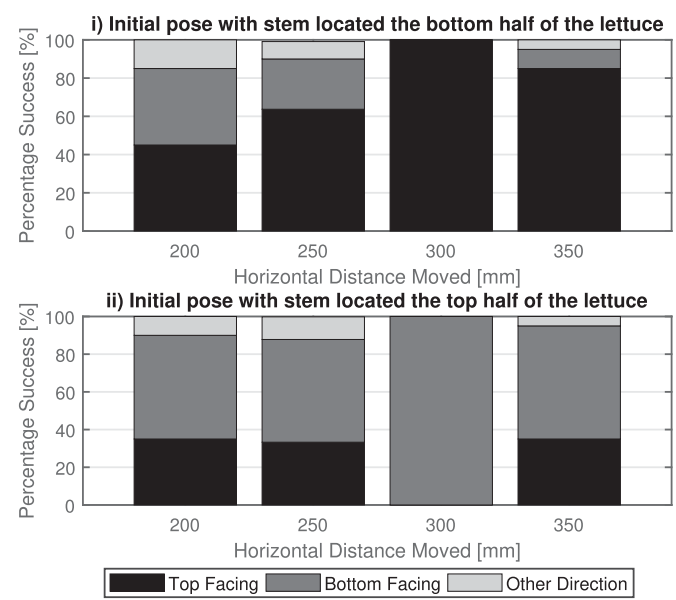

Fig. 5. Success rates when rolling a lettuce placed randomly, with the stem in the top half (top graph) or bottom half (bottom graph). 50 experiments performed using 10 lettuce.

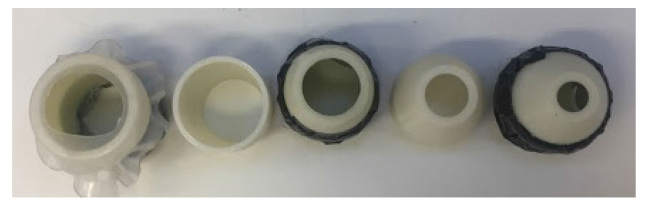

(a)
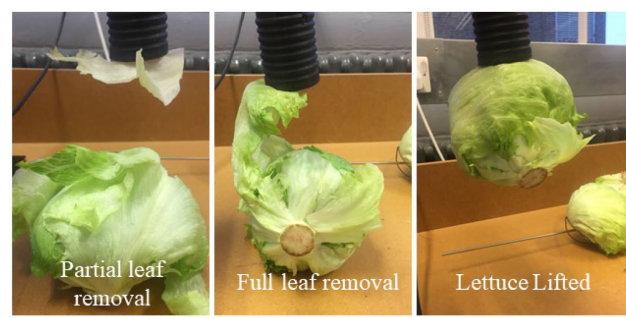

(b)

Fig. 6. (a) Nozzles tested, (b) Possible outcomes when lifting the lettuce: partial removal, full leaf removal and the entire lettuce being grasped demonstrated with a number of nozzles and without nozzle.

to allow for formation of a seal (Fig. 6(a). The normal holding force is given by $F_{h}=\Delta p A_{e}$ where $\Delta p$ and $A_{e}$ denote the pressure difference and the effective contact area respectively. Rough surfaces of the lettuce can affect the effective area in contact, reducing the holding force. Therefore a flat non-contoured area of the leaf should be used. The suction nozzle surface 
material affects the leakage flow, with compliant softer materials helping to achieve a seal. The nozzle diameter and materials must be optimized to hold and tear a single leaf opposed to lifting multiple leaves or the entire lettuce.

e) Leaf Removal point: Picking near the stem reduces the risk of tearing the fragile leaves and achieving partial leaf removal. However, the leaves are more textured (up to $4 \mathrm{~mm}$ variation in height) nearer the stem making it harder to achieve suction. Towards the edges of the leaf the thickness drops to $0.15 \mathrm{~mm}$ with the leaves more fragile, however the radius of curvature is much lower providing a flatter surface minimizing leakage flow.

f) Leaf Pulling Trajectory: The trajectory in which the leaf is moved after suction is applied affects success. Moving towards the base encourages the snapping of the leaf, as opposed to tearing. The trajectory should be optimized to maximize this snapping force.

\section{RESULTS \& DISCUSSION}

\section{A. Vision \& Detection}

The lettuce and stem detection algorithm were tested on a set of 180 pictures taken with the camera facing directly downwards above the work space at heights between $70 \mathrm{~cm}$ to $100 \mathrm{~cm}$. 10 different iceberg lettuces were used in various poses with varying light direction, light intensity and background objects (Fig. 9). In addition, 30 frames were taken after storing the produce for 3 days, inducing changes in stem color.

The algorithm had $100 \%$ detection accuracy, estimating the center with an average accuracy of $20.21 \mathrm{px} \pm 0.48 \mathrm{px}$ from the true lettuce center. The stem detection algorithm found 64 of the 79 visible stems in the data-set, with an average distance from the true stem center of $5.76 \mathrm{px} \pm 0.24 \mathrm{px}$, reaching a detection accuracy of $81.01 \%$. Given the camera height, on average we detect the lettuce true center within $7.78 \mathrm{~mm}$ and the stem within $1.73 \mathrm{~mm}$, allowing us to achieve the optimum lettuce removal point.

\section{B. Lettuce Orientation}

The lettuce was placed in a pose corresponding to a randomly generated vector with a horizontal force of $1 \mathrm{~N}$ applied at $0.1 \mathrm{~m}^{\mathrm{s}}$ using a UR5 robot arm with a soft pad, rolling the lettuce (Fig. 5). The end position of the lettuce was recorded, with the lettuce considered to be top down (such that the leaves are exposed for gripping), bottom down (stem is exposed) or in another direction. The results for 5 lettuce each rolled 10 times (Fig. 5) show that the optimum distance for horizontal rolling is $300 \mathrm{~mm}$, where for both tests the lettuce ends in one of two states, top or bottom facing. Therefore, given an initial random orientation, the lettuce should be rolled by $300 \mathrm{~mm}$. After this, stem detection should then be used to determine if the stem is exposed (bottom position) and if not, the lettuce should be rolled a further $300 \mathrm{~mm}$ in the opposite direction (to keep the lettuce within the work-space). If necessary, this process can be repeated until the top surface is exposed.
TABLE I

RESUltS FROM GRASPING LETTUCE LEAVES IN THE MidDLE OF THE LEAF AND LifTing With A Nozzle of Given DiAMETER AND MATERIAL, EACH NoZZLE TESTED ON 10 LEAVES

\begin{tabular}{cccccc}
\hline $\begin{array}{c}\text { Nozzle } \\
\text { Diameter }\end{array}$ & $\begin{array}{c}\text { Nozzle } \\
\text { Material }\end{array}$ & No Grip & $\begin{array}{c}\text { Lift } \\
\text { Lettuce (\%) }\end{array}$ & $\begin{array}{c}\text { Partial } \\
\text { Leaf } \\
\text { Tear (\%) }\end{array}$ & $\begin{array}{c}\text { Successful } \\
\text { Leaf } \\
\text { Removal (\%) }\end{array}$ \\
\hline $\mathbf{7 m m}$ & Plastic & 71 & 0 & 29 & 0 \\
$\mathbf{1 7 m m}$ & Plastic & 9 & 0 & 55 & 36 \\
$\mathbf{2 7} \mathbf{m m}$ & Plastic & 0 & 8 & 82 & 10 \\
$\mathbf{3 7 m m}$ & Plastic & 0 & 40 & 38 & 22 \\
$\mathbf{2 7} \mathbf{m m}$ & Silicone & 0 & 6 & 53 & 41 \\
$\mathbf{3 7 m m}$ & Silicone & 0 & 10 & 48 & 42 \\
\hline
\end{tabular}

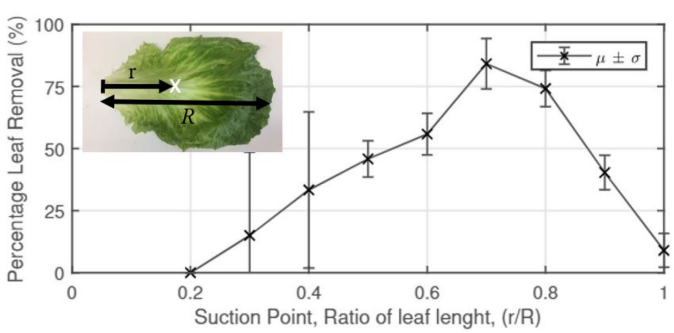

Fig. 7. Percentage area of leaf removed, with the leaf removal point along the stem of the leaf. The experiment was repeated for 10 lettuce each with two outer leaves which were removed, i.e. 20 repeats.

\section{Nozzle Design}

Nozzles of varying diameter and material (plastic or silicone) have been tested with the suction system and nozzle mounted on the end of the UR5 robot arm. A lettuce is placed in the correct 'top down' position, with the suction nozzle then lowered onto the middle of a lettuce leaf. The vacuum is then applied and the arm lifted. There are four possible outcomes: failure to grasp, lifting the entire lettuce, partial leaf removal and successful leaf removal (Fig. 6.) The results for the different nozzles are shown in Table I. The maximum lift force was measured to be $0.68 \mathrm{~N}$, significantly exceeding the typical weight of a lettuce $(\approx 0.4 \mathrm{~N})$.

The smaller nozzle ( $7 \mathrm{~mm}$ diameter), had insufficient strength to grasp any leaves, only achieving a partial tear. The largest nozzle $(37 \mathrm{~mm})$ displayed reasonable ability to remove the leaves, both partially and fully, however the increased contact area can result in lifting of the entire lettuce. The addition of a silicone outer ring to the nozzle $(27 \mathrm{~mm}$ and $37 \mathrm{~mm})$ increased the effective area, enabling a higher area of leaf tearing and successful leaf removal. The $27 \mathrm{~mm}$ silicone nozzle showed the best performance.

\section{Leaf Removal Radius}

Using the $27 \mathrm{~mm}$ nozzle from the previous experiment, lettuce leaves were gripped at varying distances along the middle of the leaf and then raised vertically, with the area percentage of leaf removed measured to the nearest $10 \%$ (Fig. 7).

Due to the extreme variability in lettuces, there is significant variation in the percentage of leaf removed, as shown by the magnitude of the error bars. At the outer edges, the leaf is fragile and tears easily, leading to a limited leaf removal. Near the stem, there is limited leaf removal due to the highly textured leaf. Thus, 


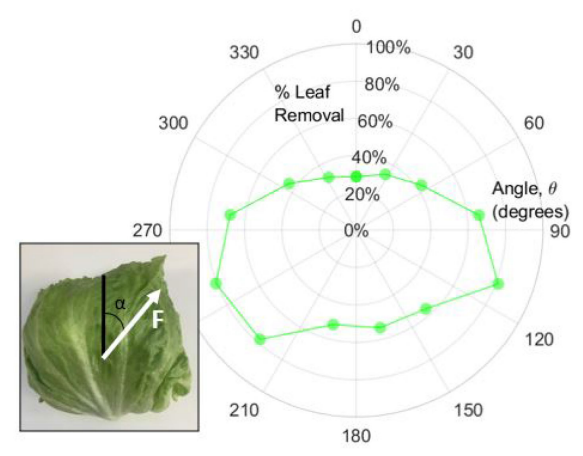

Fig. 8. Percentage of lettuce leaf removed with varying trajectory angle $\alpha$ (see inset). Each angle was tested on different outer lettuce leaves.
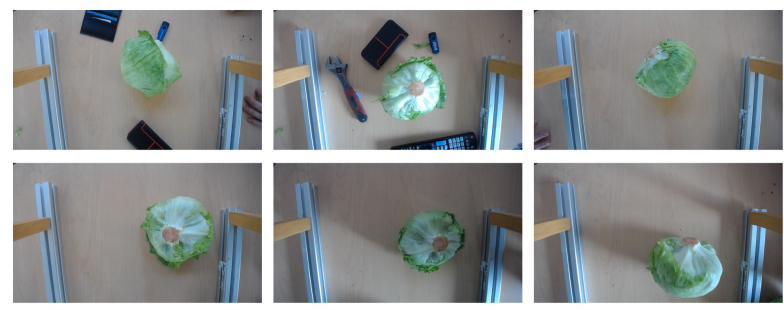

Fig. 9. Six example images in the data-set used for testing.

there exists an optimum picking location at approximately $0.7 r$ from the stem. The vision system can detect the lettuce location with an average error of $7.78 \mathrm{~mm}$, assuming a typical lettuce to have a diameter of $200 \mathrm{~mm}$, the positioning error in achieving this point is less than $5 \%$ such that successful removal can be maximized.

\section{E. Leaf Trajectory}

To test the impact of the leaf pulling trajectory, the $27 \mathrm{~mm}$ nozzle was tested $0.7 r$ along the leaf, with the arm moved at varying angles after applying suction and 'grasping' the leaf. The inclination of the trajectory relative to the normal vector of the lettuce is kept constant, at 45 degrees, such that the leaf comes clear from the body of the lettuce. The percentage area of the lettuce leaf removed is shown in Fig. 8 for different pulling angles. Pulling at 120 degrees offers the greatest chance of leaf removal as enables the leaf to snap opposed to tear.

\section{Demonstration}

To demonstrate the abilities of the methods and approaches presented, leaves were removed using the techniques discussed. Fig. 10 showcases the peeling process and videos shows the different components of the peeling process ${ }^{1}$. A lettuce was placed in a random location and orientation (as discussed previously) and is then rolled to the correct location. The second process, leaves are removed from the optimal position on the lettuce using the $27 \mathrm{~mm}$ silicon nozzle. The time for this leaf removal process (assuming the lettuce is correctly orientated) was measured to determine the average cycle time. With limited testing (10 lettuces, each with 2/3 outer leaves) there was a success rate

\footnotetext{
${ }^{1}$ Video demonstrations can be found at: https://goo.gl/S68Wnr
}

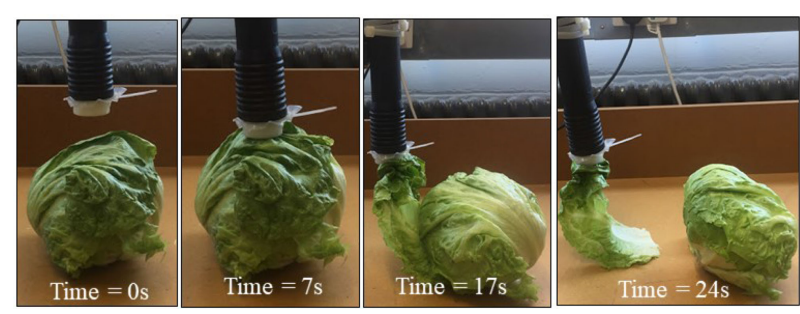

Fig. 10. Time series of the leaf removal showing the time taken to complete the process.

of full leaf peel of 50\% and partial leaf removal of 30\% with an average time of 28.5 seconds. In most cases failure was due to lifting the lettuce followed by leaf tearing.

\section{CONCLUSION}

This letter has presented mechanical and vision concepts to enable the automated removal of lettuce leaves; the proposed pipeline is the first autonomous system to perform this task. The mechanical systems have been tested and demonstrated and the required vision systems presented. Although the system has been specifically designed for the removal of lettuce leaves, there is significant wider applicability of the system and techniques developed. The model and understanding of the physical parameters affecting successful removal of lettuce leaves can be applied to many other crops such as cauliflower which is far less fragile and presents less visual variations with orientation [18]. The vision, in particular, has wide applicability in other crops where similar information would be required for the post-processing of the produce.

The lettuce and stem detection, tested on a challenging data set, have demonstrated that the proposed solutions are robust to clutter, lighting conditions, camera distance, morphological variations of the produce and changes in its position and orientation in general. The major drawback of the approach lies in the significant reliance on the produce's assumptions. Although the assumptions provide robustness, they have the potential to induce detection failures when the are not met in the real world (e.g. approximate lettuce-to-stem relative size). In this context, the tuned parameters based on the produce assumptions would need to change to reflect different produce types.

Although all components of the system have been tested (rolling, pose estimation and peeling) they are currently distinct processes. Further work to integrate this into one single end-to-end solution is required. The current system is capable of performing the peeling process with full leaf removal $50 \%$ of the times with an average time to complete of 27 seconds. Although this approach was specifically designed to minimize contact with the lettuce, reducing the chances of damage, a two 'arm' approach should be investigated ${ }^{2}$. Although not a fully automated solution, this work highlights the challenges of agri-food robotics and proposes a first step towards automating lettuce leaf peeling.

\footnotetext{
${ }^{2}$ Steps towards achieving this can be found in the Further Work video which shows lettuce peeling performed with a two arm Baxter robot. The pose estimation and peeling process is combined. https://goo.gl/S68Wnr
} 


\section{REFERENCES}

[1] L. A. Garibaldi, B. Gemmill-Herren, R. D’Annolfo, B. E. Graeub, S. A Cunningham, and T. D. Breeze, "Farming approaches for greater biodiversity, livelihoods, and food security," Trends Ecology Evolution, vol. 32, no. 1, pp. 68-80, 2017.

[2] S. Hayashi, K. Ganno, Y. Ishii, and I. Tanaka, "Robotic harvesting system for eggplants," Jpn. Agricultural Res. Quart. JARQ, vol. 36, no. 3, pp. 163 $168,2002$.

[3] T. T. Nguyen, K. Vandevoorde, E. Kayacan, J. De Baerdemaeker, and W. Saeys, "Apple detection algorithm for robotic harvesting using a RGB-D camera," in Proc. Int. Conf. Agricultural Eng., Zurich, Switzerland, 2014, pp. $1-8$.

[4] Q. Feng, W. Zou, P. Fan, C. Zhang, and X. Wang, "Design and test of robotic harvesting system for cherry tomato," Int. J. Agricultural Biol. Eng., vol. 11, no. 1, pp. 96-100, 2018.

[5] K. Kusumam, T. Krajník, S. Pearson, G. Cielniak, and T. Duckett, "Can you pick a broccoli? 3D-vision based detection and localisation of broccoli heads in the field," in Proc. IEEE/RSJ Int. Conf. Intell. Robots Syst., 2016, pp. 646-651.

[6] S. Yaghoubi, N. A. Akbarzadeh, S. S. Bazargani, S. S. Bazargani, M. Bamizan, and M. I. Asl, "Autonomous robots for agricultural tasks and farm assignment and future trends in agro robots," Int. J. Mech. Mechatronics Eng., vol. 13, no. 3, pp. 1-6, 2013.

[7] F. Yildiz, "Initial preparation, handling, and distribution of minimally processed refrigerated fruits and vegetables," in Minimally Processed Refrigerated Fruits and Vegetables. Berlin, Germany: Springer, 2017, pp. 53-92.

[8] S. Cho, S. Chang, Y. Kim, and K. An, "AE-Automation and emerging technologies: Development of a three-degrees-of-freedom robot for harvesting lettuce using machine vision and fuzzy logic control," Biosystems Eng., vol. 82, no. 2, pp. 143-149, 2002.
[9] F. Charles, P. Nilprapruck, D. Roux, and H. Sallanon, "Visible light as a new tool to maintain fresh-cut lettuce post-harvest quality," Postharvest Biol. Technol., vol. 135, pp. 51-56, 2018.

[10] C. J. Garciá, R. Garciá-Villalba, M. I. Gil, and F. A. Tomas-Barberan, "LCMS untargeted metabolomics to explain the signal metabolites inducing browning in fresh-cut lettuce," J. Agricultural Food Chemistry, vol. 65, no. 22, pp. 4526-4535, 2017.

[11] J. Rossiter and H. Hauser, "Soft robotics-The next industrial revolution," IEEE Robot. Autom. Mag, vol. 23, no. 3, pp. 17-20, Sep. 2016.

[12] C. Blanes, M. Mellado, C. Ortiz, and A. Valera, "Technologies for robot grippers in pick and place operations for fresh fruits and vegetables," Spanish J. Agricultural Res., vol. 9, no. 4, pp. 1130-1141, 2011.

[13] L. Sun, G. Aragon-Camarasa, S. Rogers, R. Stolkin, and J. P. Siebert, "Single-shot clothing category recognition in free-configurations with application to autonomous clothes sorting," IEEE/RSJ Int. Conf. Intell. Robots Syst., 2017.

[14] J. Liu, P. Li, and H. Mao, "Mechanical and kinematic modeling of assistant vacuum sucking and pulling operation of tomato fruits in robotic harvesting," Trans. ASABE, vol. 58, no. 3, pp. 539-550, 2015.

[15] C. W. Bac, J. Hemming, B. Tuijl, R. Barth, E. Wais, and E. J. Henten, "Performance evaluation of a harvesting robot for sweet pepper," J. Field Robot., vol. 34, no. 6, pp. 1123-1139, 2017.

[16] K. Kapach, E. Barnea, R. Mairon, Y. Edan, and O. Ben-Shahar, "Computer vision for fruit harvesting robots-state of the art and challenges ahead," Int. J. Computat. Vision Robot., vol. 3, no. 1/2, pp. 4-34, 2012.

[17] M. Guijarro, G. Pajares, I. Riomoros, P. Herrera, X. Burgos-Artizzu, and A. Ribeiro, "Automatic segmentation of relevant textures in agricultural images," Comput. Electron. Agriculture, vol. 75, no. 1, pp. 75-83, 2011.

[18] C. W. Bac, E. J. Henten, J. Hemming, and Y. Edan, "Harvesting robots for high-value crops: State-of-the-art review and challenges ahead," J. Field Robot., vol. 31, no. 6, pp. 888-911, 2014. 